\title{
A interpretação da prescrição sob a ótica do paciente idoso analfabeto funcional e de
}

\section{baixa escolaridade}

\author{
Interpretation of prescription from the perspective of elderly patients functional and low schools \\ Interpretación de la receta desde la perspectiva de pacientes mayores escuelas funcionales y básicas
}

Recebido: 01/30/2021 | Revisado: 02/04/2021 | Aceito: 02/06/2021 | Publicado: 02/14/2021

Richardson Lemos de Oliveira

ORCID: https://orcid.org/0000-0002-4523-2337 Universidade do Estado do Rio de Janeiro, Brasil E-mail: richardsonmedicina @gmail.com

Wilder Kleber Fernandes de Santana ORCID: https://orcid.org/0000-0001-7569-499X Universidade Federal da Paraíba, Brasil

E-mail: wildersantana92@gmail.com

Daiane de Oliveira Campos da Veiga ORCID: https://orcid.org/0000-0001-8647-2997

Universidade do Estado do Rio de Janeiro, Brasil E-mail: anyrrcampos@ hotmail.com

Aylla Monteiro Maconato

ORCID: https://orcid.org/0000-0001-7649-7840

Universidade do Estado do Rio de Janeiro, Brasil

E-mail: aylla_maconato@hotmail.com

Bruno Eduardo de Menezes Pequeno

ORCID: https://orcid.org/0000-0001-9335-8762

Universidade do Estado do Rio de Janeiro, Brasil

E-mail: bruno.eduardo@mail.com

Rebecca Rodrigues de Barros

ORCID: https://orcid.org/0000-0003-4117-2696

Universidade Federal do Rio de Janeiro, Brasil

E-mail: rebeccarbarros@hotmail.com

Lidiane Dias Reis

ORCID: https://orcid.org/0000-0001-6577-6545

Universidade Federal do Rio de Janeiro, Brasil E-mail: prof.lidianereis@gmail.com

Lívia Ferreira Pacheco

ORCID: https://orcid.org/0000-0002-6507-329X

Universidade Federal do Rio de Janeiro, Brasil

E-mail: enfliviapacheco@gmail.com

Heberth Almeida de Macedo

ORCID: https://orcid.org/0000-0003-0067-2256

Faculdade Israelita de Ciência da Saúde Albert Einstein, Brasil

E-mail: heberth-macedo@ hotmail.com

Jeanne Cristina Teixeira Gomes

ORCID: https://orcid.org/0000-0002-7557-5613

Centro Universitário Celso Lisboa, Brasil

E-mail: enfermeirajeannecristina@ hotmail.com

Isabella de Oliveira de Araújo

ORCID: https://orcid.org/0000-0003-3607-168X

Universidade Castelo Branco, Brasil

E-mail: isabellaaraujo53@gmail.com

\begin{abstract}
Resumo
A temática de nosso trabalho circunscreve discussões em torno do paciente idoso, e focalizamos o gênero prescrição, o qual é engendrado em diversas camadas das áreas da saúde. O estudo traçou como objetivo: Identificar, por meio de revisão bibliográfica, de que forma está sendo compreendida a prescrição pelo paciente idoso analfabeto funcional ou de baixa escolaridade. Foi realizada uma revisão integrativa, e os critérios de inclusão para a seleção de estudos foram selecionados apenas artigos originais em texto completo, dentro do recorte temporal de seis anos e no idioma Português Brasileiro, com resumos indexados nas bases de dados selecionadas e o recorte temporal de seis anos (2015 a 2020). Como um dos resultados principais, temos que os pacientes que frequentam as unidades de saúde com mais frequência e possuem um vínculo com o profissional, apresenta uma melhor resposta ao tratamento fazendo valer a proposta do de criar o vínculo profissional proposto pela Estratégia de Saúde da Família (ESF) e Atenção Primária a Saúde (APS).
\end{abstract}


Palavras-chave: Prescrição; Paciente idoso; Baixa escolaridade.

\begin{abstract}
The theme of our paper circumscribes discussions around the elderly patient, and we focus on the prescription genre, which is generated in different layers of the health areas. The study aimed to: Identify, through bibliographic review, how the prescription is being understood by the functional illiterate elderly patient or with low education. An integrative review was carried out, and the inclusion criteria for the selection of studies were selected only original articles in full text, within the six-year time frame and in Brazilian Portuguese, with abstracts indexed in the selected databases and the time frame six years (2015 to 2020). As one of the main results, we have that patients who attend health units more often and have a bond with the professional, present a better response to treatment, making the proposal to create the professional bond proposed by the Family Health Strategy (FHS) and Primary Health Care (PHC).
\end{abstract}

Keywords: Prescription; Elderly patient; Low education level.

\title{
Resúmen
}

La temática de nuestro trabajo circunscribe las discusiones en torno al paciente anciano, y nos centramos en el género de la prescripción, que se genera en distintos estratos de las áreas de salud. El estudio tuvo como objetivo: Identificar, mediante revisión bibliográfica, cómo la prescripción está siendo entendida por el paciente anciano analfabeto funcional o con bajo nivel educativo. Se realizó una revisión integradora y se seleccionaron los criterios de inclusión para la selección de estudios solo artículos originales en texto completo, dentro del plazo de 10 años y en portugués brasileño, con resúmenes indexados en las bases de datos seleccionadas y el plazo de seis años (2015 a 2020). Como uno de los principales resultados, tenemos que los pacientes que acuden con mayor frecuencia a las unidades de salud y tienen un vínculo con el profesional, presentan una mejor respuesta al tratamiento, haciendo la propuesta de crear el vínculo profesional que propone la Estrategia Salud de la Familia. (ESF) y Atención Primaria de Salud (APS).

Palabras clave: Prescripción; Paciente anciano; Nivel educativo bajo.

\section{Introdução}

Sabe-se que o envelhecimento é algo inevitável. Este evento ocorre de forma gradual e involuntária desde que nascemos, assim envelhecemos dia após dia. No Brasil, dados do Ministério da Saúde constatam que o envelhecimento vem ocorrendo sem que o país apresente grandes melhorias nas áreas de saúde e sociais (Ministério da Saúde, 2012). Assim, os desafios que se apresentam com a evolução do grupo dos idosos são imensos e precisam ser enfrentados visando colaborar para a manutenção do bem-estar da pessoa com 60 anos ou mais (Oliveira, 2019)

No que diz respeito à expectativa de vida no Brasil, esta tem aumentado. Num estudo realizado em 2001 por Camarano, estimava-se que a população brasileira com mais de 60 anos seja da ordem de 15 milhões de habitantes. A sua participação no total da população nacional dobrou nos últimos 50 anos; passou de 4\% em 1940 para 8\% em 1996. Projeções recentes mostram que esse segmento poderá ser responsável por quase 15\% da população brasileira no de ano 2020 [Camarano et al., 1997]. A autora ainda ressalta que esse fator se dá pela queda da mortalidade e a alta fecundidade observada nos anos 50 e 60 e à queda da mortalidade que beneficiou todos os grupos populacionais. Em suma, também devemos levar em consideração a queda da taxa de natalidade nesse recorte temporal. Outro fator que contribui para isso são as estratégias realizadas dentro da Atenção Básica.

A atenção básica caracteriza-se por um conjunto de ações de saúde, no âmbito individual e coletivo, que abrange a promoção e a proteção da saúde, a prevenção de agravos, o diagnóstico, o tratamento, a reabilitação, a redução de danos e a manutenção da saúde com o objetivo de desenvolver uma atenção integral que impacte na situação de saúde e autonomia das pessoas e nos determinantes e condicionantes de saúde das coletividades (Ministério da Saúde, 2012)

A Atenção Primária à Saúde e a Estratégia de Saúde da Família, através de seus programas, ações e estratégias, proporcionam aos seus usuários o controle de doenças crônicas como por exemplo: diabetes, hipertensão, hipotireoidismo e entre outras enfermidades crônicas não transmissíveis. No entanto, para que haja efeito, há que contar com a participação do usuário no autocuidado, seguindo à risca as informações dadas pela equipe multidisciplinar juntamente com a otimização das medicações prescritas pelo médico ou por outro profissional prescritor. 
Nesse esteio argumentativo, mediante o cenário do aumento da população idosa, delimitamos como um dos propósitos deste trabalho analisar, por meio de abordagem interpretativista, a prescrição sob a ótica do paciente idoso analfabeto funcional e de baixa escolaridade. Em nossa compreensão, na medida em que devemos nos colocar mais atentos em relação ao autocuidado inserimo-nos nesse contexto de análise da percepção por parte da população idosa. Além disso, torna-se válido mencionar que grande parte dos sujeitos da pesquisa vivem sozinhos (sem presença de familiar ou cuidador) e sem instrução letrada.

Certa vez, numa visita domiciliar, quando indagamos um usuário idoso sobre o uso de uma vitamina $\mathrm{C}$ efervescente prescrita, onde a resposta do idoso nos chamou atenção: - “...parei de tomar aquilo, faz muita espuma...” [relato do paciente], e quando foi pedido para demonstrar a forma do uso, o paciente utilizava como um comprimido de via oral ao invés de diluir em água. Tal fato, chamou a atenção dos que estavam presentes e nos fez perceber, através do uso indevido da vitamina $\mathrm{C}$ efervescente, que as comorbidades de base não se regulavam justamente pelo uso indevido das medicações prescritas ou até mesmo o não uso delas. Onde surgiu a grande preocupação: De que forma está ocorrendo a interpretação da prescrição medicamentosa pelos usuários idosos analfabetos funcionais e de baixa escolaridade?

Com base na motivação exposta através da experiencia profissional de um dos autores, o estudo traçou como objetivo: Identificar, por meio de revisão bibliográfica, de que forma está sendo compreendida a prescrição pelo paciente idoso analfabeto funcional ou de baixa escolaridade. De modo paralelo, tencionamos descrever quais estratégias podem ser adotadas pelos prescritores para melhor adesão do usuário idoso analfabeto funcional a terapia prescrita.

Para justificar o estudo, os autores buscaram abordar uma temática que é prioridade de pesquisa na Agenda Nacional de Prioridades de Pesquisa (ANPP), contido no Eixo 12 - Saúde do Idoso e em suas subagendas: 12.3 - Levantamento de metodologias inovadoras, participativas e resolutivas de educação em saúde com pessoas idosas e 12.4 - Avaliação do impacto das práticas de educação em saúde com pessoas idosas na Atenção Básica (AB).

Antes de apresentarmos s metodologia da pesquisa, explanamos uma breve discussão sobre o que seja o gênero prescrição (de medicamentos).

\section{Prescrição de medicamentos: entornos basilares}

A prescrição de medicamentos é uma atividade importante para o processo de cuidados assistenciais aos pacientes e representa ação médica fundamental. (Valadão et al, 2009) A prescrição é a transcrição do planejamento terapêutico, no formato de comandos, a serem executados pela equipe de apoio no tratamento do paciente. (Pazin- Filho et al, 2013)

Há quem aponte simplicidade no processo de comunicação envolvendo apenas 2 atores no contexto. Guzatto e Bueno (2007) diz que, a prescrição é uma ordem escrita dirigida ao farmacêutico, definindo como o fármaco deve ser fornecido ao paciente, e a este determinando em que condições o fármaco deve ser utilizado. Por outro lado, Pazin-Filho et al (2013) aponta complexidade nesse processo que é multidisciplinar, envolve médicos, farmacêuticos, nutricionistas e equipe de enfermagem, fisioterapeutas e pode adquirir enorme complexidade.

Verificar a qualidade da prescrição é avaliar a qualidade de uma parte dos serviços de saúde prestados, além de que pode ser um registro que pode indicar a satisfação do paciente, estando esta última relacionada a adesão do tratamento. Também faz parte da adesão, o entendimento da prescrição pelo paciente, pois, se este apresentar dúvida com relação ao que foi prescrito, irá sentir-se desmotivado com o tratamento, podendo alterar a prescrição segundo seu critério ou até mesmo deixar de realizá-la (Guzatto; Bueno, 2007)

É reconhecido que as prescrições têm papel ímpar na prevenção de erros de medicação e, sabe-se que tais erros podem decorrer de prescrições ambíguas, ilegíveis ou incompletas, ocasionando sérios danos ao paciente. (Valadão et. al, 2009). 


\section{Metodologia da Pesquisa}

Quanto aos aspectos teórico-metodológicos, foi realizada uma revisão integrativa de natureza qualitativa que segundo Souza, Silva e Carvalho (2010, p. 103), é a mais ampla abordagem metodológica referente às revisões, "permitindo a inclusão de estudos experimentais e não-experimentais para uma compreensão completa do fenômeno analisado". Ainda conforme as autoras, "Combina também dados da literatura teórica e empírica, além de incorporar um vasto leque de propósitos: definição de conceitos, revisão de teorias e evidências, e análise de problemas metodológicos de um tópico particular” (Souza, Silva \& Carvalho, 2010, p. 103). Quanto a natureza do estudo utilizou-se métodos qualitativos que segundo Pereira et al. (2017) são aqueles nos quais é importante a interpretação por parte do pesquisador com suas opiniões sobre o fenômeno em estudo. Neles a coleta de dados muitas vezes ocorre por meio de entrevistas com questões abertas.

Como fonte para o levantamento da produção científica foi utilizada a Biblioteca Virtual em Saúde (BVS) e os seguintes bancos de dados: MEDLINE (Literatura Internacional em Ciências da Saúde; LILACS (Literatura Latino-Americana e do Caribe em Ciências da Saúde) BDENF (Base de dados de Enfermagem). Utilizaram-se como critérios de busca as terminologias nos Descritores em Ciência da Saúde (Decs) e no Medical Subjecy Headings (MeSH): doença crônica; tratamento; saúde do idoso; educação em saúde. A ampliação na escolha almeja a redução de possíveis vieses nos estudos e ampliação nos conteúdos nesta fase da revisão integrativa.

Quanto aos critérios de inclusão para a seleção de estudos, foram selecionados apenas artigos originais em texto completo, dentro do recorte temporal de 6 anos e no idioma Português Brasileiro, com resumos indexados nas bases de dados selecionadas e o recorte temporal de dez anos (2010 a 2020). Os estudos que não atenderem os critérios de inclusão como: não se apresentarem em texto completos e originais, que se apresentarem em duplicata, estudos fora do recorte temporal, estudos que não possuírem o idioma selecionado foram excluídos mesmo que retratem questões pertinentes à temática anteriormente mencionada.

Com a combinação exata dos descritores, foram encontrados 1.537 estudos. Em texto completo 844 estudos. Somente artigos, foram encontrados 826. Após a seleção idiomática, foram delimitados 788 artigos e, ao estabelecer o recorte temporal, restaram 29 artigos. Após a leitura minuciosa, 2 foram excluídos por apresentarem em duplicata, 2 não se apresentavam em texto completo, 2 artigos estavam fora dos critérios de seleção idiomática e após a leitura do estudo na íntegra, foram excluídos 16 artigos por não se aproximarem da temática abordada na pesquisa. Por fim, restaram 7 artigos para construção da revisão integrativa. A seguir, passemos aos Resultados da coleta dos manuscritos.

\section{Resultados}

Após a seleção dos estudos com base nos critérios de inclusão e exclusão e leitura exaustiva dos manuscritos para aproximação da temática, restaram 7 artigos para caracterização do quadro e fundamentação para a construção da revisão interativa, o que pode ser verificado a seguir: 
Quadro 1: Disposição de Artigos.

\begin{tabular}{|c|c|c|c|c|c|}
\hline Título & Autores & Idioma & Periódico & Ano & País \\
\hline $\begin{array}{r}\text { Vivências de idosos } \\
\text { alcoolistas: } \\
\text { contribuiçôes para a } \\
\text { enfermagem } \\
\text { gerontológica }\end{array}$ & $\begin{array}{l}\text { Rejane de Fátima Parada } \\
\text { Viegas; Janaina Moreno de } \\
\text { Siqueira; Marilur de Donato; } \\
\text { Maria Yvone Chaves Mauro; } \\
\text { Sheila Nascimento Pereira de } \\
\text { Farias; Beatriz Gomes da } \\
\text { Silva }\end{array}$ & $\begin{array}{l}\text { Português } \\
\text { Brasileiro }\end{array}$ & $\begin{array}{l}\text { Rev enferm } \\
\text { UERJ, Rio de } \\
\text { Janeiro }\end{array}$ & 2018 & Brasil \\
\hline $\begin{array}{r}\text { Automedicação e adesão } \\
\text { ao tratamento } \\
\text { medicamentoso: } \\
\text { avaliação dos } \\
\text { participantes do } \\
\text { programa Universidade } \\
\text { do Envelhecer }\end{array}$ & $\begin{array}{l}\text { Antonio Leonardo de Freitas } \\
\text { Garcia; Andréia Naomi } \\
\text { Madoz Kaya; Eduardo } \\
\text { Antonio Ferreira; Eliana } \\
\text { Fortes Gris; Dayani Galato }\end{array}$ & $\begin{array}{l}\text { Português } \\
\text { Brasileiro }\end{array}$ & $\begin{array}{l}\text { Rev. Bras. } \\
\text { Geriatr. } \\
\text { Gerontol., Rio } \\
\text { de Janeiro }\end{array}$ & 2018 & Brasil \\
\hline $\begin{array}{r}\text { Acessibilidade ao } \\
\text { tratamento e estado de } \\
\text { saúde de pacientes } \\
\text { hemodialíticos }\end{array}$ & $\begin{array}{l}\text { Dandara Novakowski } \\
\text { Spigolon, Elen Ferraz Teston, } \\
\text { Maria Antonia Ramos Costa, } \\
\text { Edilaine Maran, Rebeca Rosa } \\
\text { de Souza, Antônio Moreira } \\
\text { Neto }\end{array}$ & $\begin{array}{l}\text { Português } \\
\text { Brasileiro }\end{array}$ & $\begin{array}{l}\text { Rev enferm } \\
\text { UERJ, Rio de } \\
\text { Janeiro }\end{array}$ & 2018 & Brasil \\
\hline $\begin{array}{r}\text { Letramento funcional } \\
\text { em saúde nos } \\
\text { portadores de doenças } \\
\text { cardiovasculares } \\
\text { crônicas }\end{array}$ & $\begin{array}{l}\text { José Antonio Chehuen Neto; } \\
\text { Lucas Assis Costa; Gabriela } \\
\text { Mazorque Estevanin; Tomás } \\
\text { Costa Bignoto; Camila } \\
\text { Isabela Ribeiro Vieira 1 } \\
\text { Frederico Afonso Rios Pinto; } \\
\text { Renato Erothildes Ferreira }\end{array}$ & $\begin{array}{l}\text { Português } \\
\text { Brasileiro }\end{array}$ & $\begin{array}{l}\text { Ciência \& } \\
\text { Saúde Coletiva }\end{array}$ & 2017 & Brasil \\
\hline $\begin{array}{r}\text { Consulta de } \\
\text { enfermagem na } \\
\text { perspectiva de usuários } \\
\text { com diabetes mellitus na } \\
\text { Estratégia de Saúde da } \\
\text { Família }\end{array}$ & $\begin{array}{l}\text { Delmo de Carvalho Alencar }{ }^{1} \text {, } \\
\text { Rosana dos Santos Costa } \\
\text { Ana Maria Parente Garcia } \\
\text { Alencar }{ }^{3} \text {, Wanderson } \\
\text { Carneiro Moreira4, Aline } \\
\text { Raquel de Sousa Ibiapina5, } \\
\text { Mária Brito de Alencar6 }\end{array}$ & $\begin{array}{l}\text { Português } \\
\text { Brasileiro }\end{array}$ & $\begin{array}{l}\text { Rev enferm } \\
\text { UFPE on line }\end{array}$ & 2017 & Brasil \\
\hline $\begin{array}{r}\text { Prevalência referida, } \\
\text { fatores de risco e } \\
\text { controle da hipertensão } \\
\text { arterial em idosos }\end{array}$ & $\begin{array}{l}\text { Chrystiany Plácido de Brito } \\
\text { Vieira; Juliete de Jesus do } \\
\text { Nascimento; Samyra } \\
\text { Sthefania Barros; Maria } \\
\text { Helena Barros Araújo Luz; } \\
\text { Andréia Rodrigues Moura da } \\
\text { Costa Valle }\end{array}$ & $\begin{array}{l}\text { Português } \\
\text { Brasileiro }\end{array}$ & $\begin{array}{l}\text { Cienc Cuid } \\
\text { Saude }\end{array}$ & 2016 & Brasil \\
\hline $\begin{array}{r}\text { Envelhecimento e } \\
\text { adesão terapêutica como } \\
\text { foco de atenção } \\
\text { educativa em } \\
\text { enfermagem no } \\
\text { intercambio estudantil } \\
\text { internacional }\end{array}$ & $\begin{array}{l}\text { Willian Alves dos Santos; } \\
\text { Ana Rita Santos Piteira; } \\
\text { Liliana Filipa Godinho Batista } \\
\text { Fernandes; Bruna Silva Leite; } \\
\text { Alberto José Barata } \\
\text { Gonçalves Cavaleiro; Geilsa } \\
\text { Soraia Cavalcanti Valente }\end{array}$ & $\begin{array}{l}\text { Português } \\
\text { Brasileiro }\end{array}$ & $\begin{array}{l}\text { Rev enferm } \\
\text { UFPE on line }\end{array}$ & 2015 & Brasil \\
\hline
\end{tabular}

Fonte: Autores.

\section{Discussão}

Segundo a Organização Mundial de Saúde (OMS), em países desenvolvidos, a adesão ao tratamento com medicamentos de uso contínuo é geralmente por volta de 50,0\%. Já em países em desenvolvimento esse valor pode ser muito menor (Garcia et al, 2018). É preciso, também, considerar que “A baixa escolaridade constitui dado preocupante na avaliação de saúde do idoso, 
pois é um dos fatores determinantes das doenças crônicas e, quanto mais baixa, de modo geral, maior a prevalência destas doenças e das complicações decorrentes da evolução das mesmas" (Vieira et al, 2016).

Ainda de acordo com Vieira et al (2016, p. 34),

o nível de escolaridade baixo é um dos mais importantes indicadores das condições de saúde da população e está intimamente relacionado à idade no Brasil, visto que muitos idosos deste século não tiveram oportunidades de estudo na juventude. Além disso, vale ressaltar que o predomínio de mulheres pode ser explicado pelo processo de feminização da velhice, devido à mortalidade masculina ser superior à feminina, especialmente nas idades avançadas, dando às mulheres maior longevidade e possibilidade de desenvolver deficiências físicas e mentais ou doenças referidas.

Ainda nesse sentido, Spigolon et al., (2018) demonstram, em seu estudo sobre o tratamento e estado de saúde de pacientes hemodialíticos, que fatores como o predomínio de idosos, sexo masculino, baixa escolaridade e fonte de renda baixa vinculada à aposentadoria e ao auxílio-doença acentuam a vulnerabilidade a qual estes indivíduos já estão expostos por conta da doença [...]. De forma paralela, Chehuen Neto et al. (2019) fazem uma breve associação entre o nível e compreensão e letramento ressaltando discrepantes resultados entre os gêneros, onde o sexo masculino apresenta-se com pequena desvantagem em relação ao sexo masculino. Os autores apontam que a escolaridade também é um determinante social com a qual o LFS mostra-se vinculado, de tal forma que os resultados deste estudo demonstraram uma relação significativa entre menor escolaridade e baixa capacidade em compreender as instruções médicas (Chehuen Neto et al. 2019)1․ Logo,

A Sociedade Americana de Insuficiência Cardíaca sugere, inclusive, que o grau de LFS do paciente seja obtido e registrado no prontuário médico, tamanha a importância desse fator na relação médico-paciente. Os profissionais da saúde, desconhecendo o grau de LFS dos seus pacientes, por vezes, utilizam uma linguagem especializada, fornecem informações insuficientes e não se certificam se foram assimiladas (Chehuen Neto et al., 2019)

Segundo o Ministério da Educação (BRASIL, 2013) o “...desafio é a alfabetização dos brasileiros maiores de 60 anos. De acordo com a pesquisa, $23,1 \%$ dos analfabetos estão nesta faixa etária.” Além disso, algumas regiões do Brasil cujo empobrecimento é maior, possuem um índice de analfabetismo perpendicular: Na pesquisa realizada no ano de 2018, eram quase 6 milhões de analfabetos com 60 anos ou mais, o que equivale a uma taxa de analfabetismo de 18,6\% para esse grupo etário (IBGE, 2018). É válido mencionar que dados da Pesquisa Nacional por Amostra de Domicílio (PNAD), por meio do Instituto Brasileiro de Geografia e Estatística indicaram que "O analfabetismo se concentra especialmente na população com mais de 60 anos e, sobretudo, na área rural, concentrado no Nordeste, no semiárido do Brasil” (PNAD, 2013).

A APPMS é um documento de caráter consultivo que além de oferecer suporte para o planejamento estratégico do Decit/SCTIE/MS e das demais áreas do MS, representa uma importante ferramenta de articulação com institutos e fundações de fomento à pesquisa, visando o estabelecimento de parcerias (públicas e privadas) para potencializar o financiamento de pesquisas em saúde e direcionar esforços para temas estratégicos e de relevância para o SUS. A estruturação da APPMS permitirá ainda o fortalecimento das ações de avaliação e monitoramento das pesquisas

\footnotetext{
${ }^{1}$ Profissionais da saúde apresentam pouca habilidade para reconhecer baixos níveis de compreensão e letramento do paciente. Buscar dialogar de uma forma mais compreensível e acessível ao paciente pode ser uma boa estratégia além de uma melhor avaliação para uma sólida percepção do grau de compreensão do usuário. Vieira et al (2016) reitera que a não adesão à terapêutica medicamentosa é identificada pelo profissional como importante problema de saúde que afeta grande número de idosos com doenças crônicas. Como consequência da não adesão ao tratamento medicamentoso podemos citar a falta de controle das enfermidades, risco aumentado de hospitalizações e aumento na mortalidade. "Há que se levar em conta que a vulnerabilidade dos idosos a efeitos adversos é muito alta, principalmente devido à necessidade de utilização de mais de um medicamento para tratamento de seus problemas de saúde, além de alterações farmacocinéticas e farmacodinâmicas que ocorrem com o próprio envelhecimento. Além disso, existe o risco de interações medicamentosas e intoxicações" (Garcia et al, 2018)
} 
financiadas no âmbito do SUS, ampliando a possibilidade de incorporação de tecnologias e dos resultados das pesquisas científicas no sistema de saúde (Ministério da Saúde, 2018).

\section{Para não sucumbir à cegueira da interpretação: a importância de atividades facilitadoras}

Ao discutirem sobre formas de comunicação em relação a pacientes idosos com hipertensão arterial, "Torna-se essencial considerar a avaliação do nível de escolaridade como fator importante para promoção de ações de atenção à saúde." (Vieira et al, 2016).

Na perspectiva de Alencar et al, 2017, quando falam sobre o modo de agir dos profissionais em relação ao paciente idoso, "É importante falar devagar, alto e olhando para ele, facilitando a comunicação pela expressão facial e leitura labial. Podese também recorrer a uma terceira pessoa quando este não consegue fornecer as informações necessárias" (Alencar et al, 2017). Garcia et al (2018) ressaltam a importância de profissionais aptos a desenvolver atividades entre a comunidade idosa e capacitados para compreensão de forma sensibilizada para que oriente os usuários de forma clara, respeitando suas limitações de compreensão ${ }^{2}$.

Há de se concordar quando Garcia et al (2018) diz que "idosos mais escolarizados tendem a compreender melhor as informações passadas pelos profissionais da saúde, além da importância relacionada ao uso racional de medicamentos [...]". Por outro lado, pacientes menos escolarizados não compreendem bem sobre as prescrições que lhes são passadas. É nesse direcionamento argumentativo que, segundo Chehuen Neto et al, essa dificuldade de compreensão das instruções em saúde nos detentores de LFS insatisfatório parece conduzir a uma ausência de autoconfiança na habilidade de praticar o autocuidado, sendo mais um fator que prejudica a adesão ao tratamento" (Chehuen Neto et al. 2019)

$\mathrm{Na}$ ótica dos autores, "destaca-se a importância do uso de linguagem simples pelos profissionais ao transmitir orientações aos pacientes, especialmente para aqueles com menor escolaridade, fator que predispõe a um LFS baixo." (Chehuen Neto et al. 2019). Desse modo,

Um regime medicamentoso complexo requer da pessoa idosa conhecimentos, capacidades e competências fundamentais na tomada de decisão, que lhes permite obter os medicamentos, administrá-los e gerir os efeitos terapêuticos e secundários de forma contínua, contribuindo positivamente para a obtenção de melhores resultados de saúde, bem-estar, qualidade de vida e adesão terapêutica (Santos et al. 2015)

Deve-se destacar que os idosos que apresentam dificuldades e pouca motivação para adesão ao tratamento precisam de esclarecimento, uma vez que a falta de instrução os impossibilita de agirem corretamente quanto ao uso e consequentemente gera retardo ao tratamento, o que requer atenção especial por parte dos profissionais (Vieira et al, 2016)

Viegas et al (2018), em seu estudo, constatam um baixo nível de escolaridade entre os participantes, pacientes no grupo de alcoolismo. Em seu estudo, os autores correlacionam o alcoolismo às pessoas de menor escolaridade ${ }^{3}$. Além disso, os pacientes

\footnotetext{
${ }^{2}$ Outro fator apresentado nas pesquisas é a falta de conhecimento do paciente sobre a sua doença crônica, no qual evidenciou que a compreensão das condições cardiovasculares esteve associada ao grau de LFS, visto que mais da metade $(64,1 \%)$ dos pacientes com letramento não adequado referiu não compreender sua doença plenamente (Chehuen Neto et al. 2019). Outros [...] “...90\% dos entrevistados não conheciam seu tipo de diabetes e muitos sequer sabiam da existência de mais de um tipo da doença. Não saber tal informação, apesar de interpretado como dado não relevante porque todos possuíam diabetes tipo 2, demonstra desconhecimento dos usuários em relação à doença e tratamento." (Alencar et al, 2017)

${ }^{3}$ Sendo assim, segundo Chehuen Neto et al. (2019) pacientes com déficit de numeramento deve ser interpretados como sujeitos que encontram dificuldades para manejar os horários de suas medicações propostas pelo plano terapêutico. O autor afirma também que para que haja sucesso no tratamento e melhor qualidade e atenção a saúde o usuário necessita ter um bom compreendimento sobre suas doenças e seu estado de saúde. Mediante tal fato, entende-se que o paciente com LFS baixo possui dificuldades de assimilar informações fundamentais para a continuidade através do autocuidado. Outro fato importante a terapia medicamentosa necessita de auxílio, como a mudança nos hábitos de vida concomitantes com a otimização dos fármacos, no entanto, nos resultados da pesquisa de Spigolon, et al. (2018) muitos afirmaram que mudanças nos hábitos diários, como a dietética, a hídrica e a laboral, lhes incomodavam, o que mostra que essas mudanças alimentares, limitações nas atividades físicas, lazer, trabalho, a utilização contínua de medicações [...].

Outro fator que sobressaiu nos resultados de Garcia et al (2018), que a presença de companheiro, marido, cônjuge, contribui positivamente no cuidado, visto que na maioria dos casos, analisados em seu estudo, a adesão ao tratamento, auxílio para os horários da administração dos
} 
que apresentam um Letramento Funcional (LSF) em Saúde deficitário, apresem inúmeras dificuldades de entendimento sobre medicações. Diante de tais averiguações, concordamos com Chehuen Neto et. al sobre o fato de que "É necessário que se desenvolvam medidas educativas para que os profissionais de saúde possam ajustar sua linguagem, receituários e outros instrumentos de comunicação às necessidades e competências dos pacientes mais susceptíveis a déficits no LFS." (Chehuen Neto et al, 2019)

A educação em saúde, incluindo tópicos que envolvam o uso de medicamentos e o autocuidado, deveria ser inclusa na formação de adultos, bem como de todas as profissões da saúde independente da sua relação com esta tecnologia (Garcia et al, 2018). Deste modo, reitera-se que a educação em saúde se configura como prática prevista e realizada pelos profissionais que compõem a equipe de saúde da família, em que são abordados temas como medidas de controle da HA, orientações sobre alimentação, atividade física e uso correto dos medicamentos. E de que forma profissionais da saúde podem estar contribuindo metodologicamente, ou seja, em sua práxis laboral? Por meio da "[...] realização de estratégias educativas que venham a reforçar o esclarecimento da população acerca da doença, ou seja, seus tipos, seus sinais e sintomas, como se dá seu controle, desmistificando tabus, dentre outras informações[...]" (Alencar et al, 2017)

Corroboramos, por fim, com Vieira et al (2016), os quais reiteram a real necessidade de fortalecimento das políticas públicas de promoção da saúde e prevenção de doenças, bem como entendemos de extrema importância o posicionamento de Spigolon, et al., que atestam a necessidade de incorporar no planejamento estratégias de cuidado se concretiza a fim de responder às reais necessidades dos pacientes idosos (Spigolon, et al. 2018)

\section{Considerações Finais}

Todas as amostras selecionadas apresentaram peculiaridades sobre a compreensão do paciente analfabeto funcional ou de baixa escolaridade sobre a prescrição. Inicialmente, pode-se concluir que a escolaridade interfere diretamente na otimização das medicações por parte do usuário. Além disso, os estudos apontam que os profissionais de saúde não apresentam sensibilidade a essa questão, ou seja, subnotificam ou não levam em conta a escolaridade como fator determinante para uma melhor resposta terapêutica, pode-se destacar também que nos casos de falta de vínculo com o paciente esse fator deficitário apresenta-se com mais dificuldade de ser notado pelo profissional ou exposto pelo próprio paciente.

Tal problemática atua como barreira fazendo com que o paciente não exponha suas dúvidas e fragilidades sobre o plano terapêutico ou até mesmo quanto ao seu estado de saúde, não reconhecendo sequer suas doenças de base. Por outro lado, vale ressaltar que pacientes que frequentam as unidades de saúde com mais frequência e possuem um vínculo com o profissional, apresenta uma melhor resposta ao tratamento fazendo valer a proposta do de criar o vínculo profissional proposto pela Estratégia de Saúde da Família (ESF) e Atenção Primária a Saúde (APS).

Nota-se que a pesquisa apresenta como propostas, estratégias de Educação em Saúde através de dinâmicas de grupo, grupos de educação em saúde para redução das dificuldades de compreensão e abordagem de outras temáticas, porém, nenhuma das pesquisas apresentaram de forma detalhada suas estratégias para tal fato. É de suma importância ressaltar que os profissionais de saúde busquem desenvolver o hábito de perguntar o nível de escolaridade durante a consulta e de indagar o paciente, pedindo que o mesmo repita a explicação sobre o uso otimizado de suas medicações, tal feito, possibilitará o profissional de reconhecer ser o paciente compreendeu de fato a explicação sobre o uso regular de suas medicações. Não podemos negar a importância da

mesmos e até o acompanhamento com melhor frequência aos serviços de saúde. Logo, pôde-se observar no estudo de Alencar et al (2017) que a menor adesão ao tratamento de suas enfermidades e frequência nos serviços de saúde são de pessoas viúvas. Assim, "O maior tempo de acompanhamento na unidade básica de saúde é importante, pois possibilita o vínculo entre usuário e equipe de saúde, facilitando a interação e o sucesso das práticas. Tal fato viabiliza maior proximidade do profissional de saúde com a população e a ênfase em ações focalizadas sobre os grupos de risco e fatores de risco comportamentais permitem prevenir o aparecimento ou a manutenção de doenças e comorbidades" (Vieira et al, 2016) 
realização dos grupos de educação em saúde, porém, o profissional tem o papel ser resolutivo ao identificar o déficit do paciente no primeiro momento.

Contudo, baseado nas evidências que as buscas nos trouxeram, sugere-se a sensibilização e estimulação de profissionais da saúde para desenvolver interesses pela temática, visando ampliar as discussões sobre tal assinto no meio científico. Desta forma, almejamos reduzir as fragilidades da população exposta e melhorar as condições de vida aos pacientes.

Estima-se que a pesquisa ofereça base sólida e consistente para a transparência e construção de conteúdo exposto, assim como pretende-se que os resultados deste estudo possam ampliar a produção de novos conhecimentos, a fim de que estes possam ser utilizados para contribuir na realização de mais pesquisas na área da Saúde Pública, principalmente na Saúde do Idoso e Saúde Coletiva.

\section{Referências}

Alencar, D. C., Costa, R. S., Alencar, A. M. P. G., Moreira, W. C., Ibiapina, A. R. S., \& Alencar, M. B. (2017). Nursing consultation in the perspective of users with diabetes mellitus in the family health strategy. Revista de Enfermagem UEPE On line (REUOL). Recife - Brasil.

Brasil. Ministério da Saúde (2006). Portaria nº 2.528 de 19 de outubro de 2006. Aprova a Política Nacional de Saúde da Pessoa Idosa. Brasília.

Brasil. Ministério da Saúde (2012). Política Nacional da Atenção Básica (PNAB). Brasília.

Brasil. Ministério da Saúde (2013). Diretrizes para o cuidado das pessoas com doenças crônicas nas redes de atenção à saúde e nas linhas de cuidado prioritárias. Brasília.

Brasil. Ministério da Saúde (2017). Política Nacional da Atenção Básica (PNAB). Brasília.

Brasil. Ministério da Saúde (2018). Agenda de Prioridades de Pesquisa do Ministério da Saúde. Brasília.

Brasil. Presidência Da República (2019). LEI No 8.080, de 19 de setembro de 1990. Brasília.

Brasil. Ministério da Educação (2018). Analfabetismo no país cai de 11,5\% para 8,7\% nos últimos oito anos. Pesquisa em: http://portal.mec.gov.br/component/tags/tag/34167.

Brasil. Agência IGBE Notícias (2019). PNAD Contínua 2018: educação avança no país, mas desigualdades raciais e por região persistem. Pesquisa em: https//agenciadenoticias.bge.gov.br/agencia-sala-de-imprensa/2013-agencia-de-noticias/releases 24857 -pnad-continua-2018-educacao-avanca-no-pais-mas-desigualdades-raciais-e-por-regiao-persistem.

Camarano, A. A. (2010). Cuidados de longa duração para a população idosa: um risco social a ser seguido. Rio de Janeiro, IPEA.

Camarano, A. A. (2002). Envelhecimento da População Brasileira: Uma contribuição Demográfica. Texto para a discussão nº 858, RJ: IPEA.

Camarano, A. A. (2004). Os novos idosos brasileiros. Muito além dos 60? IPEA.

Camarano, A. A. (2016). Living longer: Are we getting older or younger for longer? Vibrant: Virtual Brazilian Anthropology. 13(1), Brasília

Chehuen Neto, J. A., Costa, L. A., Estevanin, G. M., Bignoto, T. C., Vieira, C. I. R., Pinto, F. A. R., \& Ferreira, R. E. (2017). Functional Health Literacy in chronic cardiovascular patients. Ciências \& Saúde coletiva.

Garcia, A. L. F., Kaya, A. N. M., Ferreira, E., A., Gris, E., F., \& Galato, D. (2018). Self-medication and adherence to drug treatment: assessment of participants of the Universidade do Envelhecer (the University of Aging) program. Revista Brasileira de Geriatria e Gerontologia. Brasil.

Guzatto, P., Bueno, D. (2007). Analisis of drug prescriptions dispensed by a pharmacy in a basic health unit. Rev HCPA. Brasil.

Oliveira, A. S. (2019). Transition, epidemiological transition and population aging in Brazil. Revista Brasileira da Geografia Médica e da Saúde.

Pazin-Filho, A. Frezza, G., Matsuno, A. K., Alcântara, S. T., Cassiolato, S., Bitar, J. P. S., Pereira, M. M., \& Fávero, F. (2013). In-Hospital prescription guidelines for undergraduate medical students. USP Medicina (Ribeirão Preto).

Pereira, A. D., Shitsuka, D. M., Parreira, F. J., \& Shitsuka, R. (2018). Metodologia da pesquisa científica. UAB/NTE/UFSM.

Santos, W. A., Piteira, A. R. S., Fernandes, L. F. G. B., Leite, B. S., Cavaleiro, A. J. B. G., \& Valente, G. S. C. (2015). Aging and therapeutic adherence as care focus on education in nursing international student exchange. Revista de Enfermagem UEPE On line (REUOL). Recife - Brasil.

Spigolon, D. N., Teston, E. F., Costa, M. A. R., Maran, E., Souza, R. R., \& Neto, A. M. (2018). Accessibility to treatment and health status of hemodialytic patients. Revista de Enfermagem UEPE On line (REUOL). Recife - Brasil.

Souza, M. T., Silva, M. D., \& Carvalho, R. (2010). Revisão integrativa: o que é e como fazer. Einstein. 8(1), $102-106$.

Valadão, F. A., Moreira, A. L. P., Andrade, L. C., Pires, C. A., Firmino, K. F., \& Brum, C. A. (2009). Prescription medical: focus on errors in prescriptions. Rev. Bras. Farm., 90(4): 340-343. 
Research, Society and Development, v. 10, n. 2, e25410212494, 2021

(CC BY 4.0) | ISSN 2525-3409 | DOI: http://dx.doi.org/10.33448/rsd-v10i2.12494

Vieira, C. P. B., Nascimento, J. J., Barros, S. S.; Luz, M. H. B. A., \& Valle, A. R. M. C. (2016). Prevalência referida, fatores de risco e controle da hipertensão arterial em idosos. Cienc Cuid Saúde.

Viegas, R. F. P., Siqueira, J. M., Donato, M., Mauro, M. Y. C., Farias, S. N. P., \& Silva, B. G. (2018). Lives of alcoholic older adults: contributions to gerontological nursing. Rev Enferm UERJ, Brasil. 